\title{
Persistent ST-segment elevation following pericardiocentesis: caution with thrombolytic therapy
}

\author{
H.H. Hsia, N.H. Kander and M. J. Shea \\ Division of Cardiology, Department of Internal Medicine, University of Michigan Medical Center, Ann Arbor, Michigan, USA \\ Received: 15 January 1987; accepted: 17 January 1987
}

\begin{abstract}
We report a case of persistent electrocardiographic ST-elevation following pericardiocentesis despite lack of evidence for transmural infarction or vasospasm. The electrocardiographic pattern was felt to reflect subepicardial injury due to a small myocardial laceration. The implications of this finding are discussed.
\end{abstract}

Key words: Pericardiocentesis - Complications of myocardial infarction - Thrombolysis

Emergency pericardiocentesis for electromechanical dissociation is performed as a potential lifesaving maneuver for patients in whom pericardial tamponade or hemopericardium may be present. Frequently, clinical proof of these conditions may be absent yet pericardiocentesis is performed empirically because tamponade is a "reversible" cause of electromechanical dissociation. However, pericardiocentesis is not without risk. Complications range from vasovagal reactions and transient arrhythmias to pulmonary edema, pneumothorax and hemopericardium $[1,2]$.

We report a case of a patient developing a new focal injury pattern on the electrocardiogram following pericardiocentesis for electromechanical dissociation. This was unrelated to infarction or vasospasm but was most likely due to a myocardial laceration. The importance of this observation relates to the rapidly changing treatment of acute myocardial infarction with the emphasis on venous lytic therapy and new interventional strategies.

\section{Case report}

A 66-year-old woman with longstanding rheumatoid arthritis, rheumatic mitral stenosis and paroxysmal atrial fibrillation was admitted to hospital for worsening congestive heart failure. The patient's heart failure was felt to be related to verapamil used to control the atrial fibrillation. Previous echocardiographic evaluation had demonstrated thickened anterior and posterior mitral valve leaflet echoes with parallel movement, a diminished E-F slope, left atrial enlargement, and normal left ventricular function without pericardial effusion (Fig. 1).

On the third hospital day, the patient suffered a witnessed cardiac arrest. Cardiopulmonary resuscitation was started immediately. The initial rhythm was ventricular fibrillation which subsequently converted to a pulseless idioventricular rhythm with cardiopulmonary resuscitation. The possibility of cardiac tamponade was entertained in view of the electromechanical dissociation. Therefore, two pericardiocenteses were attempted via the subxiphoid approach with a 20 -gauge spinal needle aimed toward the left shoulder. The results were nonproductive for any fluid or blood and the needle was quickly removed. No V-lead electrocardiographic monitoring was used and no rhythm disturbance was noted during the procedure.

Subsequently the patient converted to normal sinus rhythm with a blood pressure of $110 / 80 \mathrm{mmHg}$. The immediate post-arrest electrocardiogram showed new $1.5 \mathrm{mV}$ ST-elevation in leads V4-5 (Fig. 2).

Because of post-arrest chest pain and in view of the electrocardiographic findings suggestive of myocardial injury, it was felt that the patient could be suffering an acute myocardial infarction. An emergency cardiac catheterization was performed which revealed: mean right atrial pressure $8 \mathrm{mmHg}$; right ventricular pressure $39 / 6 \mathrm{mmHg}$; pulmonary artery pressure $30 / 15$, mean $22 \mathrm{mmHg}$; mean pulmonary capillary wedge pressure $16 \mathrm{mmHg}$; estimated mitral valve area (Gorlin) $1.7 \mathrm{~cm}^{2}$, and cardiac index $2.91 / \mathrm{min} / \mathrm{m}^{2}$. Left ventriculography revealed normal 


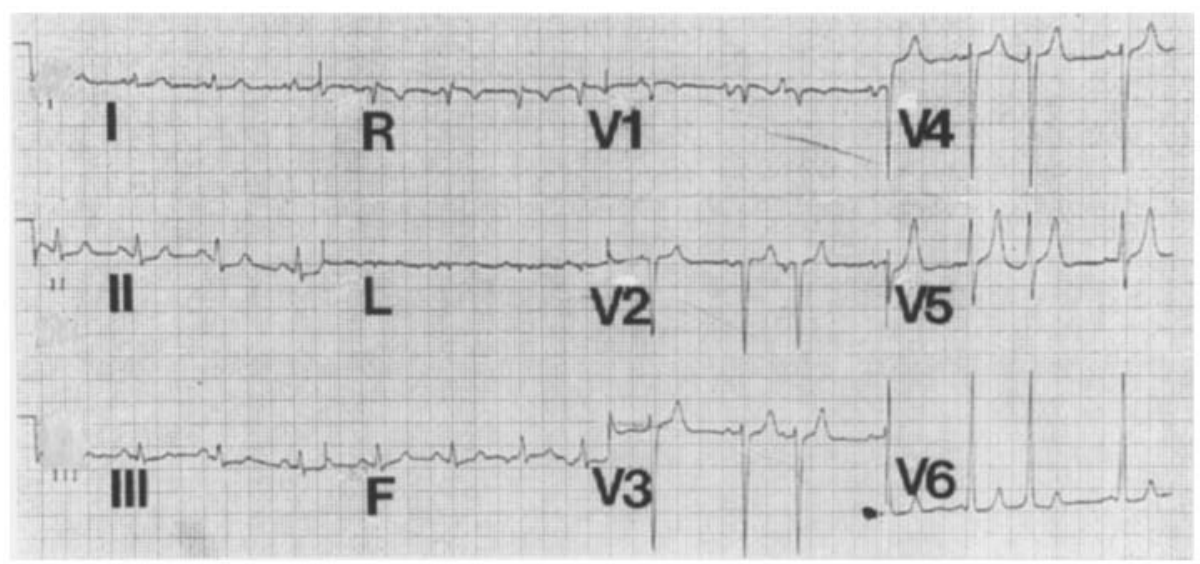

Fig. 1. The admission electrocardiogram demonstrates sinus rhythm, poor $\mathbf{R}$ wave progression, prominent precordial voltage and atrial premature contractions segmental wall motion and overall normal left ventricular contraction with mild mitral regurgitation and a calcified mitral valve. Coronary arteriography revealed a normal left dominant circulation without evidence of stenosis or vasospasm. Within $24 \mathrm{~h}$ the creatine kinase rose to 2300 I.U. with $12 \%$ MB fraction present. The electrocardiographic injury pattern persisted for $24 \mathrm{~h}$ but had completely resolved by $48 \mathrm{~h}$. A technetium pyrophosphate scan was obtained $72 \mathrm{~h}$ post-arrest, no focal uptake was documented. A repeat echocardiogram showed no pericardial effusion and no wall motion abnormalities.

\section{Discussion}

In 1956, Bishop et al. [3] described the electrocardiographic changes during pericardiocentesis. The "current of injury" is detected as either ST-segment elevation or PR-segment elevation when the ventricular or atrial epicardium is in contact with the needle. This observation has been used as a safeguard for inadvertent myocardial injury; nevertheless, myocardial laceration without electrocardiographic changes have been well documented $[4,5]$.
The present case is unusual because following pericardiocentesis the injury pattern was observed to be localized to $V_{4}-V_{5}$ and this persisted for over $24 \mathrm{~h}$, yet there was no complementary evidence for transmural injury. The electrocardiogram subsequently returned to the baseline appearance with preservation of R-wave voltage without evidence of " $Q$ " waves. Ventriculography and echocardiography did not reveal wall motion abnormalities and coronary arteriography during the ST-elevation revealed no evidence for vasospasm or significant coronary atherosclerosis. The rise in creatine phosphokinase suggests myocardial damage occurred but the technetium scan did not reveal focal uptake nor was there evidence for a diffuse increased uptake. While post-cardioversion myonecrosis cannot be excluded, we feel that the relatively focal injury pattern with negative technetium scan and elevated enzymes are most consistent with a small area of subepicardial injury and necrosis from the pericardiocentesis needle. This is supported by the direction of the pericardiocentesis attempts: from the subxiphoid to left shoulder regions. While the persistence of ST-segment elevation remained unexplained, it is doubtful that transmural injury occurred since there

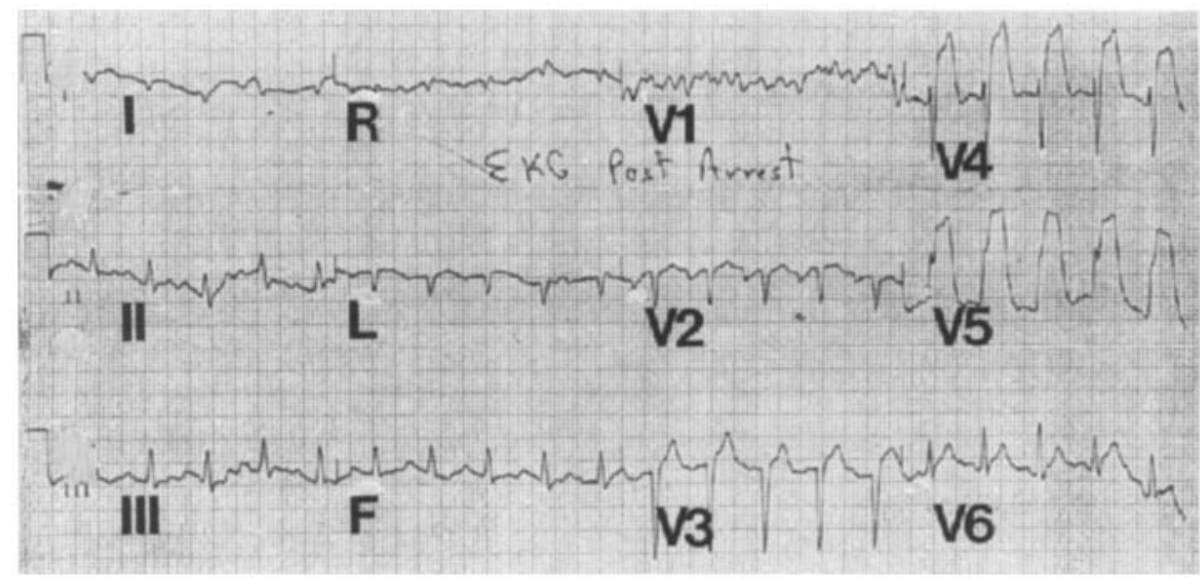

Fig. 2. Electrocardiograph immediately after postresuscitation shows $1.5 \mathrm{mV}$ ST-elevation in leads $\mathrm{V}_{4-5}$, which persisted during cardiac catheterization and did not resolve until $48 \mathrm{~h}$ later 
was normal regional wall motion at the time of repeat echocardiography and the technetium scan was also normal.

Persistent elevation of the ST-segments following pericardiocentesis may have important implications for management. Such electrocardiographic changes are usually felt to reflect subepicardial injury as seen most commonly with acute coronary occlusion due to thrombosis or vasospasm. Because myocardial salvage is clearly a time-dependent process, current therapeutic strategies have increasingly centered on administration of thrombolytic therapy via the intravenous route rather than the intracoronary route. This is in part related to timing and in part related to practicality and cost. While our patient had a relative contraindication to lytic therapy, viz., cardiopulmonary resuscitation, many clinicians would be inclined to pursue therapy in such a situation. In view of the case presented, a persistent focal electrocardiographic injury pattern following pericardiocentesis should raise concerns about whether there is additional, concordant evidence for myocardial injury. Independent confirmation of ischemic injury should be established via echocardiography or, in some cases, coronary arteriography and ventriculography. The risk of empiric lytic therapy in such a setting is unknown but with the potential complication of myocardial laceration could be catastrophic.

Acknowledgement. We thank Lisa Hackbarth for excellent secretarial assistance.

\section{References}

1. Miller JI (1986) Surgical management of pericardial disease. In: Hurst JW (ed) The Heart. McGraw-Hill, New York, p 2009

2. Wong B, Murphy J, Chang CJ, Hassenein K, Dunn M (1979) The risk of pericardiocentesis. Am J Cardiol 44:110

3. Bishop LH, Estes EH, McIntosh HD (1956) The electrocardiogram as a safeguard in pericardiocentesis. JAMA 62:264

4. Sobel SM, Thomas HM, Evans RW (1975) Myocardial laceration not demonstrated by continuous electrocardiographic monitoring occurring during pericardiocentesis. N Engl J Med 282:1222

5. Gueron M, Hirsch M, Wanderman K (1975) Myocardial laceration not shown by ECG during pericardiocentesis. N Engl J Med 293:938

Dr. M. J. Shea

Division of Cardiology

University of Michigan Medical Center

1500 East Medical Center Drive

3910 B Taubman Center

Ann Arbor, MI 48109-0366

USA 